\title{
Erratum to: Le prélèvement de tissu adipeux : un acte médical pour la recherche clinique. Perspectives pour le soin courant
}

\section{Erratum to: Adipose tissue biopsy: a useful medical act in clinical research. Prospects in medical care}

\author{
L. Genser · C. Vatier $\cdot$ M. Keophiphath $\cdot$ J. Aron-Wisnewsky $\cdot$ C. Poitou $\cdot$ K. Clément $\cdot$ J.-P. Bastard \\ (C) Springer-Verlag France 2014
}

Erratum to: Obésité DOI 10.1007/s11690-013-0403-8

Une erreur est intervenue concernant le nom d'un des auteurs cet article : il fallait lire M. Keophiphath (et non M. Keophyphath).

\footnotetext{
L. Genser $\cdot$ C. Vatier $\cdot$ J. Aron-Wisnewsky $\cdot$ C. Poitou $\cdot$ K. Clément $\cdot$ J.-P. Bastard $(\bowtie)$ Institut de cardiométabolisme et nutrition, ICAN, hôpital Pitié-Salpêtrière, Paris

e-mail : jean-philippe.bastard@tnn.aphp.fr

L. Genser

Assistance Publique-Hôpitaux de Paris, (AP/HP), hôpital Pitié-Salpêtrière,

service de chirurgie digestive hépato-bilio-pancréatique et transplantation hépatique, 75013 Paris

C. Vatier $\cdot$ J. Aron-Wisnewsky $\cdot$ C. Poitou $\cdot$ K. Clément

Assistance Publique-Hôpitaux de Paris (AP/HP), hôpital Pitié-Salpêtrière, service de nutrition, 75013 Paris

C. Vatier · J.-P. Bastard

Inserm, Université Pierre et Marie-Curie-Paris 6, UMRS_938, CDR Saint-Antoine, 75012 Paris

M. Keophiphath

AdipoPhyt SAS, 75013 Paris

J. Aron-Wisnewsky $\cdot$ C. Poitou $\cdot$ K. Clément

Inserm, Université Pierre et Marie-Curie-Paris 6,

UMRS Nutriomique U872 Equipe 7, Centre de recherche des Cordeliers, 75006 Paris

J.-P. Bastard

Assistance Publique-Hôpitaux de Paris (AP/HP), hôpital Tenon, service de biochimie et hormonologie, 75020 Paris
} 